livraisons

d'Histoire

de l'Architecture

\section{Livraisons de l'histoire de l'architecture}

14 | 2007

Piscines

\title{
Bains-Baden, les piscines bruxelloises
}

Isabelle Pauthier

\section{OpenEdition}

Journals

Édition électronique

URL : http://journals.openedition.org/lha/426

DOI : $10.4000 /$ /ha. 426

ISSN : 1960-5994

\section{Éditeur}

Association Livraisons d'histoire de l'architecture - LHA

\section{Édition imprimée}

Date de publication : 10 décembre 2007

Pagination : 55-67

ISSN : 1627-4970

\section{Référence électronique}

Isabelle Pauthier, «Bains-Baden, les piscines bruxelloises », Livraisons de l'histoire de l'architecture [En ligne], 14 | 2007, mis en ligne le 10 décembre 2009, consulté le 01 mai 2019. URL : http:// journals.openedition.org//ha/426 ; DOI : 10.4000//ha.426

Ce document a été généré automatiquement le 1 mai 2019.

Tous droits réservés à l'Association LHA 


\title{
Bains-Baden, les piscines bruxelloises
}

\author{
Isabelle Pauthier
}

La signification sociale de la pratique de la baignade a connu de fortes évolutions dans l'histoire de la culture occidentale. Au XIX' siècle, les divertissements et les loisirs restent réservés à la noblesse et à la bourgeoisie dont ils constituent un signe distinctif. Après la première guerre mondiale, ils prennent progressivement de l'importance dans le quotidien de toutes les classes sociales. Les établissements de bain traditionnels qui allient bassin de natation, bains, douches, hydrothérapie et parfois salle de gymnastique résultent de la volonté des pouvoirs publics de promouvoir l'hygiène corporelle et la promotion de la santé auprès des classes laborieuses dont les logements ne sont pas équipés en eau courante. Ces préoccupations laissent progressivement place à la pratique sportive et récréative. Les bains traditionnels s'adaptent dans les années 1930 à la concurrence d'un type inédit d'établissements, les solaria, qui mettent en avant la pratique sportive de plein air. Ceux-ci ont aujourd'hui disparu, à l'inverse des bains traditionnels, dont certains sont particulièrement remarquables sous l'angle architectural et décoratif. À partir des années 1950, chaque commune bruxelloise se dote d'une piscine dans un objectif d'éducation sportive. À part l'exemple notable du Neptunium à Schaerbeek et de la piscine Longchamp à Uccle (C. De Meuter et J. Koning, architectes, 1965), la qualité architecturale laisse le plus souvent place à la fonctionnalité ${ }^{1}$

\section{Conditions de vie et progrès social}

2 La révolution industrielle s'est développée précocement en Belgique. Elle place les ouvriers dans des conditions d'existence difficiles: allongement constant du temps de travail, bas salaires, conditions d'existence a minima. À Bruxelles, la densité augmente spectaculairement après l'indépendance du pays (1830). Entre 1830 et 1890, la population du centre de Bruxelles passe ainsi de 90.000 à 159.000 habitants. À partir du milieu du XIX e siècle, les pouvoirs publics prennent conscience du caractère insupportable de 
l'entassement et les courants philanthropiques établissent les bases de l'amélioration des conditions de vie. Il s'agit, par la prophylaxie, de conserver et de reproduire la force de travail. Les bases théoriques de la démocratisation de la culture du bain apparaissent au XVIII ${ }^{\mathrm{e}}$ siècle dans le cadre du mouvement hygiéniste. Médecins et pédagogues font la preuve de l'influence bénéfique de l'eau sur la santé. La purification du corps à l'aide d'immersion et d'aspersion se propage comme moyen efficient pour améliorer les conditions sanitaires des ouvriers et les fortifier grâce à différentes pratiques d'hydrothérapie et de natation. ${ }^{2}$ L'hygiène publique est perçue au début du $\mathrm{XX}^{\mathrm{e}}$ siècle comme « une manifestation les plus certaines du progrès moderne ${ }^{3}$ ». « La foi en l'hygiène est générale ${ }^{4}$ » « Allez au bain, ça fait du bien ${ }^{5} »$.

La seconde moitié du XIX ${ }^{e}$ siècle voit fleurir nombre d'initiatives destinées à encourager l'application des principes d'hygiène: l'assainissement des quartiers ouvriers, la construction des premiers logements sociaux, l'aménagement d'un réseau public de distribution d'eau potable dans les années 1850 ou encore la création, sur l'initiative du ministre des affaires intérieures Charles Rogier, de divers organismes dont la mission est d'améliorer l'hygiène publique : le service central de santé et d'hygiène, le conseil supérieur d'hygiène et les comités de salubrité. Pendant l'épidémie de choléra de 1850, le ministre adresse une missive aux gouverneurs de province pour promouvoir la construction de bains publics sur le modèle anglais. Les idées novatrices relatives à l'hygiène corporelle se répandent également grâce à des journaux comme "La Santé" ", distribué à partir de 1849, et aux travaux du congrès général d'hygiène qui s'est tenu à Bruxelles en 1851-1852. Les bains y sont investis d'un rôle moral : ils contribuent aux valeurs sociales et à l'ordre moral. Ils assouplissent les membres et contribuent à une éducation virile, les « exercices natatoires ${ }^{6}$ » étant généralement réservés aux garçons.

\section{Une œuvre de propagande hygiéniste}

4 Victor Boin, président du comité olympique, milite dans les années 1930 pour que la natation soit rendue obligatoire dans les écoles. Certains complexes scolaires bruxellois modèles se dotent dès leur création d'un bassin de natation, à l'instar de la piscine de l'école de la rue de la Ruche, construite en 1907 par l'architecte Henri Jacobs (1864-1935)7. Victor Boin écrit : «En 1890, quelques sportsmen aux idées avancées, désireux de rendre populaire un exercice salutaire et pratique, fondèrent la première société belge de natation. Ils la baptisèrent: " Cercle de natation de Bruxelles " et commencèrent dans notre pays une propagande qui resta longtemps nulle et sans effets heureux. [...] Avant 1880, on ne nageait pas en Belgique ou, du moins, si l'on nageait, c'était à l'encontre de toute méthode et d'une façon désordonnée ${ }^{8}$ ". La natation ne devint un sport reconnu que lorsque le cercle des régates de Bruxelles créa sa section de natation, puis en 1897, grâce à la fondation du « Brussels Swimming Club ».

\section{Les Bains économiques, rue des Tanneurs, 1854}

Pendant l'épidémie de choléra de 1848-1850, le prolifique architecte Wynand Janssens (1827-1913) propose aux autorités communales la construction de bains dans le quartier populaire des Marolles. Il s'inspire des «bath and waterhouses» anglais. La façade des Bains économiques, sobre et rigoureusement symétrique évoquait un petit établissement scolaire. Elle était organisée autour des entrées séparées pour les hommes et les femmes. 
Le prix d'accès modique fixé à 20 centimes - le salaire moyen d'une heure de travail s'élevait à 30 centimes - contribua au succès de ces installations. Les bains économiques furent utilisés jusqu'à la première guerre mondiale puis furent démolis en 1953 pour laisser place à des logements sociaux construits par le Foyer bruxellois (cité de la Querelle).

\section{Le Bain royal, rue du Moniteur, 1879}

Construite par la société anonyme du Bain royal, c'est la première piscine couverte de Bruxelles. Comme son nom l'indique, cet établissement se voulait prestigieux et s'adressait à un public bourgeois attiré par le faste et la représentation sociale. Il comprenait une piscine, des bains individuels, des bains thérapeutiques, un atrium, un jardin, un café... L'architecte Adolphe Vanderheggen (1839-1906) déploya dans un certain luxe des matériaux modernes comme le fer et le verre. La coupe du Bain royal (ill. 1) évoque le dispositif architectural centré des halles métalliques doté d'une charpente elliptique ${ }^{9}$.

\section{1 : Le Bain royal}

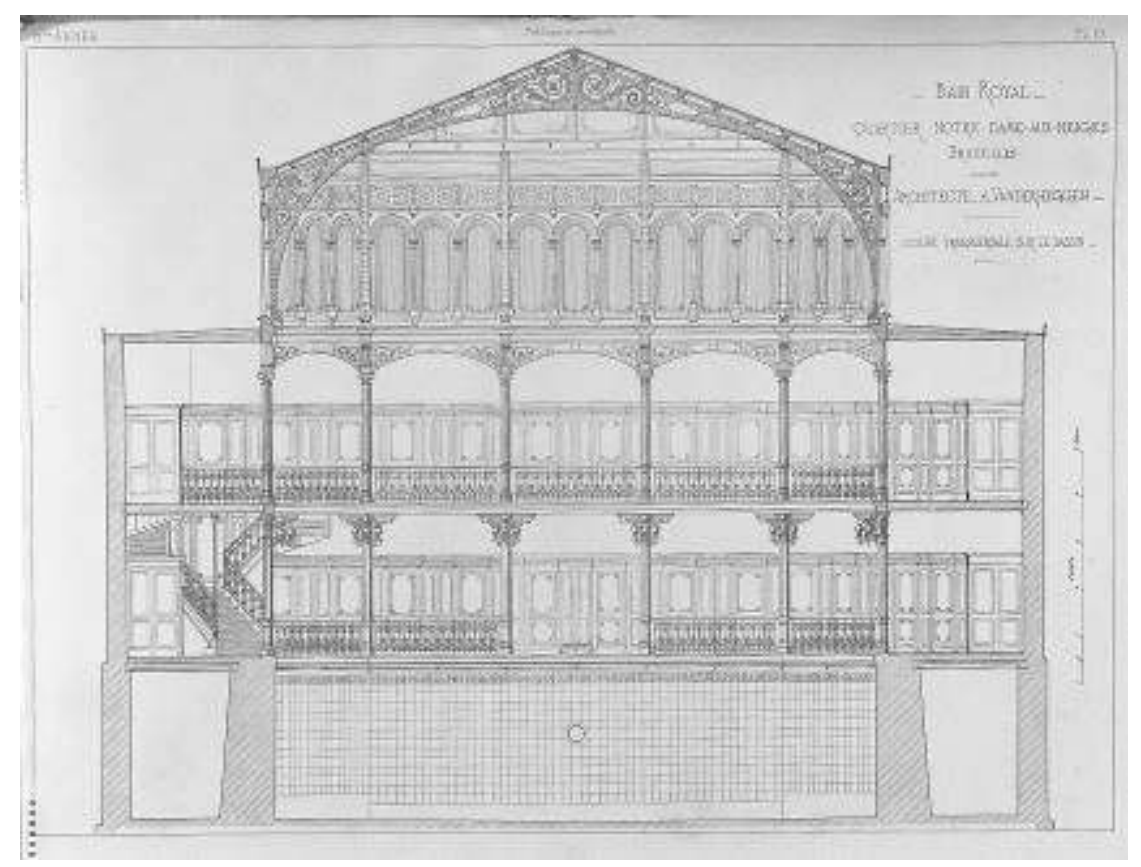

\section{COUPE TRANSVERSALE SUR LE BASSIN}

CL. ARCHIVES D'ARCHITECTURE MODERNE, BRUXELLES

Il n'y a pas encore de circuit élaboré qui permette de séparer la circulation des pieds chaussés et des pieds nus. L'intérieur a été complètement démoli en 1969 pour réaliser une école. La façade, qui donne sur la rue de l'Enseignement, présente encore les motifs décoratifs qui évoquent la destination originelle du bâtiment: poissons, coquilles, hippocampes. 


\section{Les complexes de bains communaux au tournant du $\mathrm{XX}$ e siècle}

Plusieurs communes bruxelloises prennent l'initiative d'édifier des complexes de bains publics assortis de piscines couvertes dans des quartiers ouvriers. Le prix d'entrée était compris entre 20 et 50 centimes. Les bains turcs étaient plus coûteux, jusqu'à 2 francs.

La piscine d'Ixelles, construite 10 rue de la Natation par les architectes Jules Rau (1854-1923) et Alexandre Cooreman (1880- ?), est la plus vieille piscine existante en région bruxelloise. Elle établit une typologie d'organisation qui restera en vigueur jusque dans les années cinquante : l'avant-corps est séparé du bassin, une galerie abrite les cabines. Sa construction fit l'objet d'un concours d'architecture en janvier 1899. La piscine comprend un bassin de natation avec lavabos, douches et cabines mais était dépourvue de bains turcs. La façade en briques est soulignée par des bandeaux en pierre bleue et est animée par des carreaux de céramique blanche, Jules Rau ayant un intérêt particulier pour les recherches chromatiques sur les matériaux. L'armature métallique apparente, les colonnes en fonte dotées de chapiteaux corinthiens, la balustrade en fer forgé aux dessins géométriques préservent en grande partie l'aspect d'origine malgré une rénovation lourde en 2000.

10 Les bains de Saint-Gilles, 38 rue de la Perche, réalisés entre 1902 et 1905 par les mêmes architectes présentaient un programme architectural identique. En 1938, l'architecte Inghelbrecht fut chargé d'agrandir la piscine en lui ajoutant deux étages de cabines et la toiture d'origine fut remplacée par un toit escamotable sur une structure en béton armé. Les bains douches ont été supprimés mais les bains turcs sont toujours en fonction et possèdent leur association de défense. Les carreaux en faïence blanche et jaune et les robinetteries et mains courantes en bronze poli sont d'origine. Les chaudières au charbon ont été adaptées au mazout en 1958 puis au gaz naturel en 1972. Le centenaire de la piscine a coïncidé avec l'installation $185 \mathrm{~m}^{2}$ de panneaux solaires sur la toiture.

\section{L'évolution de la pratique sociale du bain dans l'entre- deux-guerres : de l'hygiène à la détente}

11 À l'instar du cinéma, le sport et le jeu deviennent des loisirs très populaires dans l'entredeux-guerres. L'exercice physique est valorisé et l'adage « un esprit sain dans un corps sain » répond à l'aspiration de libération du mouvement moderne. Le sport et l'hygiène corporelle, d'abord objets d'injonction, deviennent une pratique sociale répandue. Les sportifs incarnent les héros populaires de l'entre-deux-guerres.

L'écrivain Paul Werrie, dans un article du premier numéro de la revue Bâtir, en 1933, stigmatise Bruxelles qu'il considère, au regard des villes allemandes «qui, toutes, possèdent leurs bains aquatiques et solaires", comme une "ville croupissante ». Il dénonce la piètre qualité des eaux de baignade, qualifiée par lui de «bouillon de culture ». Il souligne que le congrès d'oto-rhyngologie de Paris stipule que l'eau des établissements de bain doit être filtrée, désinfectée et renouvelée quotidiennement, conditions qui sont loin d'être remplies à Bruxelles. Les dispositions sanitaires imposeront bientôt que les cabines doivent être à deux entrées et désinfectées chaque jour, les douches et brossages préalables des pieds au savon doivent être obligatoires. Les 
usagers se plieront bien volontiers à ces contraintes de salubrité. L'aération est fondamentale. Les contraintes liées à l'hygiène forcent ainsi l'évolution des programmes architecturaux.

\section{Les bains de Saint-Josse, 21-23 rue Saint-François, 1930-1933}

13 Les bains communaux sont construits selon les plans des architectes Joseph Bytebier (1880-1941) et Charles Schaessens (1880-1953) à l'emplacement d'anciennes impasses ouvrières expropriées en 1913 dans le cadre d'un projet d'assainissement. Ils sont associés avec un complexe de logements sociaux.

La façade des bains s'étage le long d'une rue en pente. Elle se compose de trois niveaux de huit travées rythmées au niveau des étages par des pilastres reposant sur un puissant socle en pierre bleue percé de petites ouvertures. L'élévation est en brique jaune et en pierre blanche dite de France. L'appareil de briques est animé de motifs de chevrons. La conception architecturale se réclame de l'Art déco utilitaire, animé de formes géométriques et empreint d'une certaine monumentalité. De grandes baies en plein cintre éclairent le rez-de-chaussée dans cette rue étroite. Les ouvertures rectangulaires des étages sont jumelées ou en agencées en triplet. L'inscription «Bains-Baden » est gravée dans la pierre en lettrage Art déco. Le bâtiment est couronné par une corniche saillante interrompue par deux pignons triangulaires à tympan ajouré (ill. 2).

\section{2 : Le Neptunium}

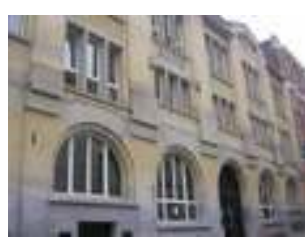

FAÇADE SUR LA RUE

CL. DE L'AUTEUR.

15 Les parties centrale et droite du bâtiment abritent le bassin de natation et les bains turcs. La partie gauche, plus sobre, correspond au caractère humble des bains individuels. Des entrées séparées desservent ces deux départements, reprenant le modèle d'organisation considéré comme idéal dans la littérature spécialisée. Un dispositif de sas sépare la circulation pieds nus et pieds chaussés. La piscine est couverte d'une voûte en berceau reposant sur des arcs de béton en anse de panier. À la retombée de cette voûte, les parties supérieures des murs sont percées de larges baies vitrées sous lesquelles court une galerie fermée. Un étage de cabines donne, au niveau du premier étage, sur une galerie périphérique, l'autre, au niveau du rez sur le bord étroit du bassin. Les éléments fonctionnels constituent le support d'une sobre décoration. Le hall d'entrée monumental est dallé de marbre tacheté noir et blanc. La cuve du bassin est carrelée de faïences blanches, bleu clair et foncé et noir.

Les bains seront inaugurés en 1934. À l'époque, on espérait que les habitants prendraient au moins un bain par mois car la préoccupation sanitaire reste primordiale. Dans le cadre du débat sur la longueur du bassin, l'architecte met l'accent sur le fait que le bassin se doit de répondre prioritairement à des buts d'hygiène plutôt qu'à des objectifs sportifs. 
L'ensemble du complexe a largement gardé son aspect d'origine (ill. 3) hormis quelques interventions malheureuses réversibles (portes de cabines en formica, gardes corps en verre fumé, statue de naïade en plastique).

\section{3 : Bains Saint-Josse}

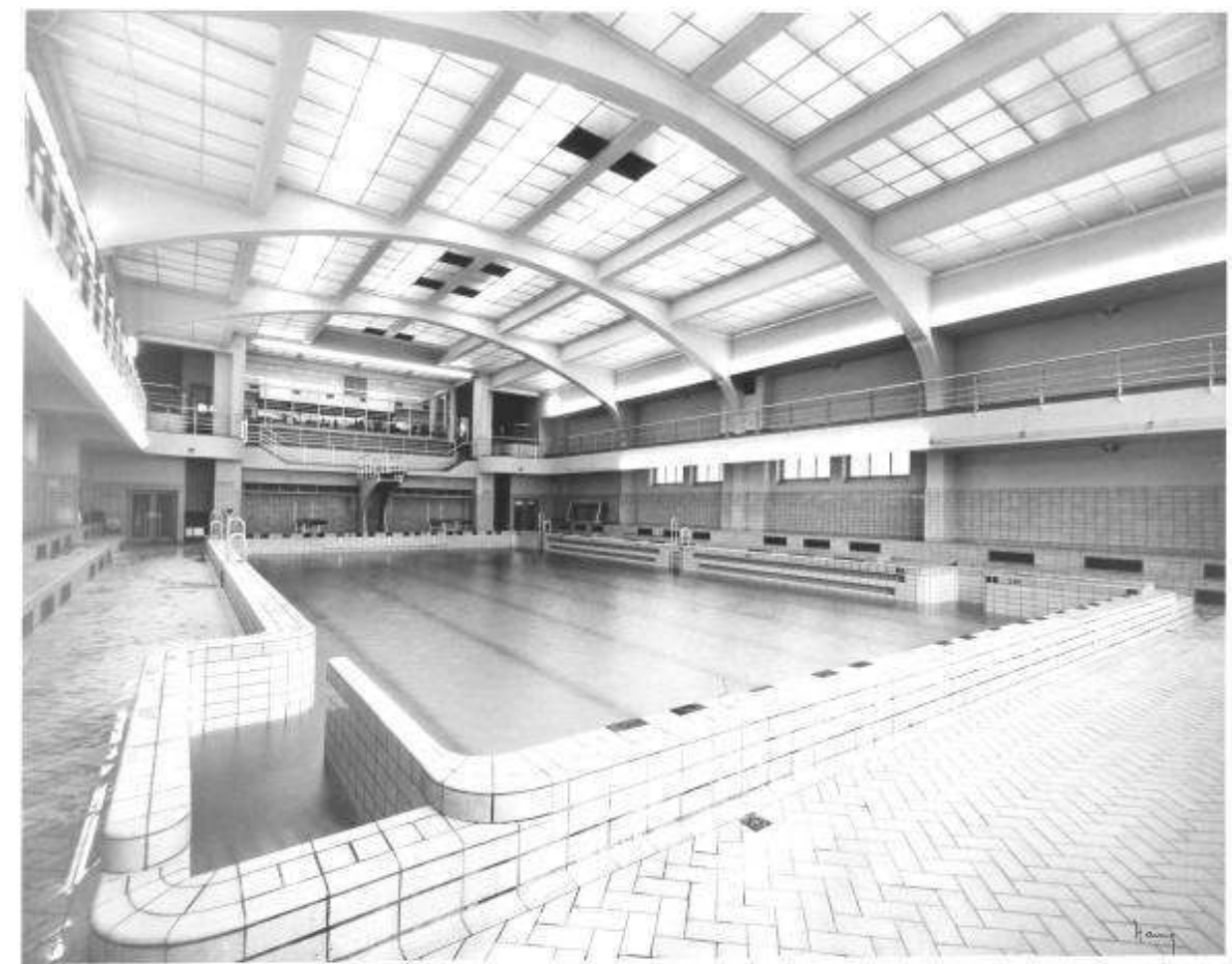

VUE DU gRAND BASSIN

CL. ARCHIVES D'ARCHITECTURE MODERNE, BRUXELLES

Des panneaux de mosaïque représentant un paysage aquatique ont été rajoutés en 1967-68.

Comme la plupart des bassins bruxellois qui présentent un déficit d'exploitation de 375.000 euros en moyenne $\mathrm{e}^{10}$, leur situation financière fut très critique à la fin des années 1990. Les bains douches et les bains turcs furent abandonnés en 1975. Les bains douches furent remis en service en 2005 sur l'initiative d'une association d'aide aux sans-abri. La piscine est défendue comme outil d'intégration dans ce quartier populaire à forte population immigrée.

"Qui construit un solarium, supprime un hôpital "11

Durant l'entre-deux-guerres, plusieurs solaria furent aménagés à Bruxelles dans plusieurs communes de l'agglomération. Il s'agit de grandes piscines en plein air assorties de plaines de jeux, de terrasses et de buvettes qui mettaient l'accent sur la détente et le sport. Les tabous traditionnels comme la séparation des espaces réservés aux hommes et aux femmes disparaissent. Ils illustrent l'évolution de la pratique de la baignade qui n'a plus pour objectif central l'hygiène mais acquiert une fonction sportive et récréative.

Le plus célèbre est le solarium Van Schelle, construit à Saint-Gilles en 1932, rue de la Glacière, sur l'initiative du champion de natation Martial Van Schelle selon les plans des architectes Léon Emmanuel Govaerts (1891-1970) et Alexis Van Vaerenbergh (1885-?). 
Dans les années soixante, la mode de la baignade en plein air est oubliée et ces solaria ont fermé les uns après les autres. Ils ont tous été démolis.

\section{L'après-guerre}

21 Plusieurs complexes de bains sont édifiés à Bruxelles dans les années cinquante sur base des principes d'avant-guerre dans leur conception. Ce sont les derniers exemples où la piscine est couplée à des bains individuels.

\section{Les Bains du centre, 28 rue du Chevreuil, 1950-1953}

22 Après la construction des Bains économiques en 1854, plusieurs projets sont développés afin d'étendre l'offre sanitaire dans le quartier populaire des Marolles. En 1949, l'architecte Maurice Van Nieuwenhuyse (1891-1964) réalise le projet le plus moderne et le plus spectaculaire de l'époque. Il s'agit du premier bassin dans lequel les cabines de déshabillage sont séparées des bains. La galerie sert aux spectateurs. Les travaux se révèlent particulièrement laborieux en raison de la qualité du sous-sol sablonneux dans lequel affleure un véritable lac souterrain.

La façade largement vitrée constitue un manifeste moderniste dont la verticalité est encore accentuée par des hampes de drapeau. Un large portique ouvert met l'entrée en valeur. L'architecte s'est inspiré de la piscine de la Sauvenière à Liège, réalisée par Georges Dedoyard ${ }^{12}$ en 1940, qui comprend deux bassins superposés. Compte tenu du poids de l'eau et de l'instabilité du sous-sol, ce parti architectural constitue une prouesse technique. La structure portante des deux bassins, réalisée en béton, est indépendante de la structure du bâtiment. Les réservoirs d'eau et les installations d'épuration se trouvent dans les caves et l'eau était pompée vers les bassins dans un circuit fermé. Les bassins sont éclairés par une gigantesque verrière à l'arrière qui donne vue aux nageurs sur les toits du quartier (ill. 4). 


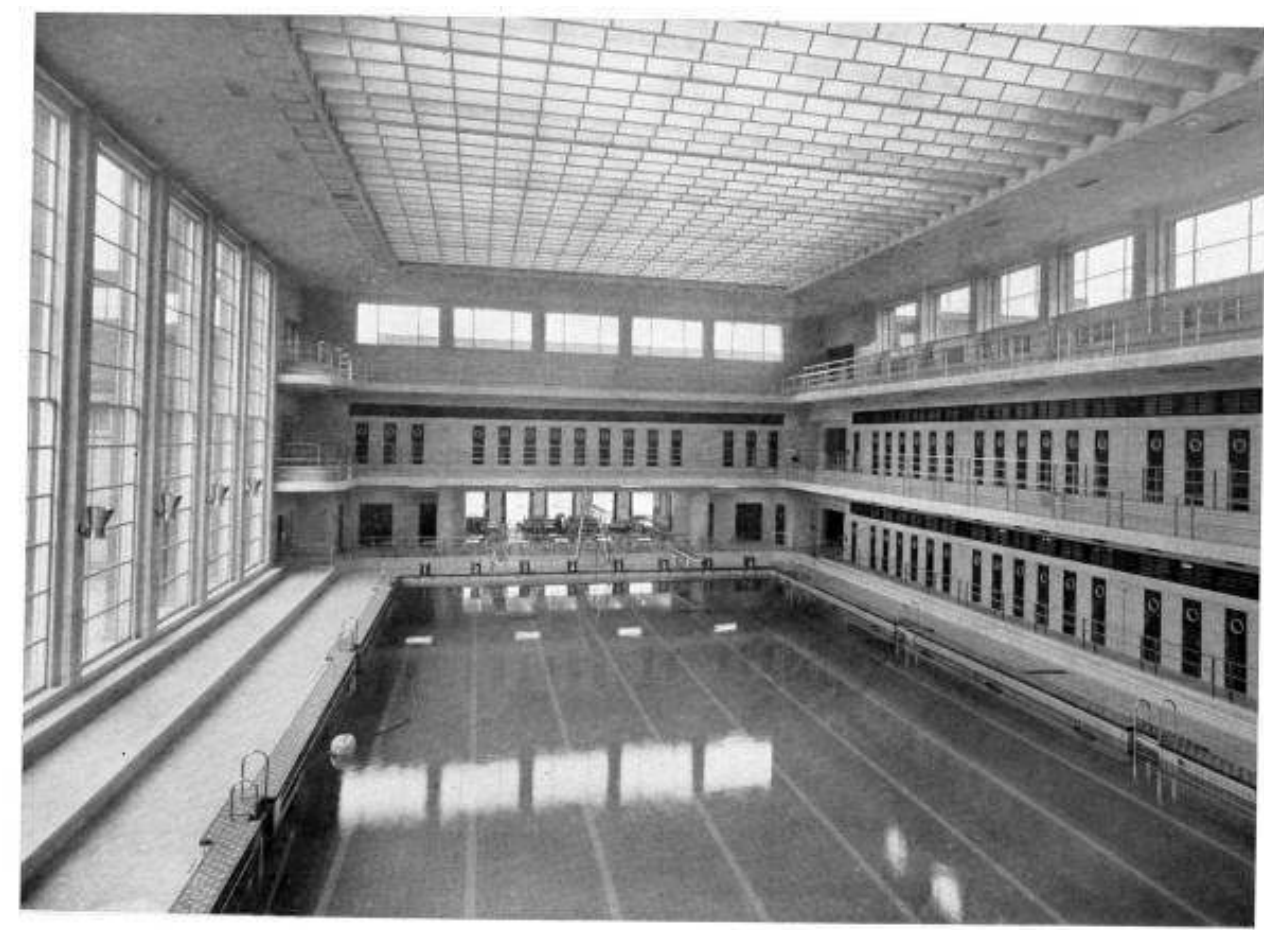

CL. ARCHIVES D'ARCHITECTURE MODERNE, BRUXELLES quatorze douches et baignoires pour hommes et dames. Le premier étage accueille les bureaux d'administration, le gymnase, les vestiaires scolaires, les lavatorys et la piscine scolaire de 18 mètres sur 10 qui comprend d'étonnants bords surélevés. Le deuxième étage abrite un salon de coiffure et de manucure, un dispensaire, le logement du concierge. La grande piscine de natation est un vaisseau de $650 \mathrm{~m}^{2}$ sur double hauteur ( 9 mètres de haut) constitué par de puissants arcs en béton armé de 19 mètres de portée, sur lesquels reposent des poutres longitudinales qui soutiennent les parois verticales au plafond vitré disposé en gradins. Une galerie réservée aux spectateurs prolonge l'espace de la buvette. Le bassin présente des dimensions de 25 mètres par 12. Il est équipé de plages latérales garnies de gradins destinés au public des compétitions. La plupart des revêtements de sol est d'origine. 


\section{Neptunium, 56 rue de Jérusalem, 1950-1957}

27 Le Neptunium constitue avec les Bains du centre un témoignage majeur de l'architecture sportive moderniste de l'après-guerre. C'est la plus grande piscine bruxelloise de l'époque. Elle se distingue par sa "grande liberté formelle et d'organisation ${ }^{13}$ et la qualité de son éclairage. C'est également la première piscine dotée d'un système à pulsions d'air pour désembuer le hall des bassins.

L'architecte est Laurent Senterre, architecte communal, probablement rompu aux programmes utilitaires. Le terrain mis à disposition est situé au centre de la commune, à proximité des écoles et des principales voies de communication. Il présente une superficie de près d'un hectare, ce qui a permis d'éviter des travaux importants de terrassement puisque aucun sous-sol n'a été creusé. La construction repose sur des pieux Franki. Elle se compose deux blocs séparés par un joint de dilatation : un bloc en façade, un à l'arrière. Cette façade de style moderniste affirme son caractère novateur. La vocation rationnelle et sociale $\mathrm{du}$ mouvement moderne s'exprime dans la simplification des formes, la géométrisation des volumes, des arrêtes soulignées, la volonté de rendre accessible à tous la beauté et la rigueur.

La façade monumentale en parement clair est étirée sur un socle en granit bleu du Hainaut, dit « pierre bleue » en Belgique. Elle présente une symétrie stricte relativement imposante et comprend trois niveaux et dix-sept travées. La façade des étages est divisée en trois, un corps central sur deux niveaux largement vitré et légèrement en surplomb et deux parties latérales symétriques un peu plus basses. Le corps central, composé de sept travées étroites, arbore des pilastres de section carrée auxquels sont fixées des hampes de drapeaux qui confèrent à cette section une verticalité qui contraste avec le reste de la façade. Les cinq portes d'entrée en métal occupent une position centrale. Un bandeau horizontal relie les trois éléments de la façade et porte l'inscription bilingue «BainsBaden ». La façade se caractérise par sa planéité, elle est totalement dépourvue de relief autre que celui que confère le jeu des volumes. Elle est percée de larges ouvertures placées dans le plan de la façade et munies de châssis métalliques.

Les auteurs de projets ont voulu valoriser le caractère ornemental de l'édifice en organisant un concours ouvert à tous les artistes belges en juillet 1954. Le concours fixait que le programme décoratif devait valoriser les bienfaits de l'élément aquatique et de la natation. Le premier prix fut attribué à une sculpture en cuivre réalisée par Stan Hensen qui représente deux sirènes qui se détachent devant une grande mosaïque destinée au mur principal du hall. Cette mosaïque réalisée par Géo de Vlamynck ${ }^{14}$ mesure 15 mètres de long sur 2,70 mètres de haut. Le groupe de deux sirènes en ronde bosse en cuivre repoussé se détache au centre. La dynamique de ce groupe est rendue par leurs bras levés qui appellent le public à jouir des joies de la natation. Un fond composé de larges champs colorés évoque les reflets bleus, verts et mordorés de l'océan, rendus par l'alternance de tesselles mates et brillantes. La composition se lit de droite à gauche. À l'extrémité droite se trouve un groupe de trois naïades. L'une, alanguie, énonce les joies de la baignade. Ses deux compagnes, assises dans une posture qui évoque les demoiselles d'Avignon, tournent la tête vers le groupe de nageurs situés à l'autre extrémité de la composition. L'une d'elle abrite sous son bras un hippocampe, évocation du nom de l'établissement. Deux nageurs abandonnés aux ondes, attirés par le champ de sirènes, sont portés par une vague. Les tesselles en pâtes de verre de quatre centimètres sur quatre proviennent de 
Murano. Des espaces transitionnels en tesselles blanches délimitent les contours des figures et leur confèrent relief et lisibilité ${ }^{15}$.

Le toit de la piscine était prévu en béton armé mais en 1950, le conseil communal a décidé de construire un toit en verre et en métal afin de permettre l'éclairage naturel du bassin. Le hall, qui comprend les deux bassins, s'élève sur quatre niveaux de galeries. Des gradins sont réservés aux spectateurs. Une paroi latérale vitrée de 28 mètres sur 12 s'ouvrait au sud sur un solarium qui a été englobé en 1996 avec des châssis en P.V.C.

Le bassin mesure 33,33 mètres de long sur 16 mètres de large, dimensions qui correspondaient aux normes olympiques de l'époque. Il est recouvert de carreaux lisses bleu pâle, les plages sont en carreaux de grès beige. La profondeur s'étale entre un et trois mètres, la partie la plus profonde du bassin se situe à l'avant, face à la cafétéria. Le bassin d'apprentissage se situe sous un plafond bas qui lui confère une certaine intimité qui contraste avec l'ampleur de l'espace dévolu au grand bassin. Ses escaliers sont interrompus par une plate forme rehaussée par une sculpture en granit poli de René Harvent (1925-2004) qui figure une otarie.

Dès l'entrée, la qualité des matériaux employés s'impose. Le hall est couvert de dalles de marbre rose serties par des joints en laiton. Les poignées des portes d'entrée représentent le sceptre de Neptune, les bouches d'aération propagent de l'air chaud au travers de lyres situées sur des grilles dont les ondulations rappellent les flots. Des supports en formes de vaguelettes supportent les mains courantes des escaliers. Le style architectural évoque immanquablement l'architecture des grands paquebots transatlantiques de l'entre-deuxguerres, en particulier avec la référence au bastingage pour la balustrade en métal et l'usage des hublots qui décorent les portes des cabines et les parois du bassin. Le grand bassin est entouré de deux terrasses. Le deuxième étage est réservé à une vaste salle de culture physique de $200 \mathrm{~m}^{2}$ assortie de vestiaires et de douches. Les bains douches ont été désaffectés puis remplacés par des saunas, bancs solaires et bains à bulles. Le complexe comprenait également un salon de coiffure, un salon de pédicure et une infirmerie dans la mesure où un examen médical était obligatoire dans les compétitions de polo et de natation.

La piscine a été dotée en 1995 d'équipements récréatifs comme un toboggan pour répondre à la concurrence des centres sportifs. Le Neptunium a été menacé de fermeture définitive en 1979 et en 2002. Cette menace a suscité une mobilisation importante des habitants et des associations de protection du patrimoine ${ }^{16}$. La piscine a finalement été rénovée pour un montant de deux millions d'euros avec l'aide de la Région, qui édicte les normes d'hygiène relatives aux bassins de natation et de l'État. La commune a récemment fait nettoyer la façade dans le cadre d'un programme de revitalisation urbaine.

Les bains publics des années 1950 présentent, outre les bassins de natation, des bains individuels destinés à l'hygiène. Une part non négligeable des habitations bruxelloises est à cette époque encore dépourvue de sanitaires. Dans les années 1960 et 1970, la construction de bassins de natation s'accélère. Ils sont prioritairement orientés vers le sport et les loisirs et non plus vers les soins du corps et l'hygiène.

\section{Un patrimoine à prendre en compte}

36 L'histoire des bains bruxellois apporte une contribution importante à l'histoire des conceptions architecturales et à l'histoire sociale d'une grande ville. Les piscines 
répondent aux besoins pédagogiques des écoles, les bains aux besoins d'hygiène des populations les moins favorisées et les bains turcs au besoin de délassement d'une population plus aisée. L'évolution de ces complexes d'hygiène puis de détente illustre le changement de signification de la pratique sociale de la baignade et de la natation. La diffusion des installations sanitaires dans les logements a fait perdre de leur utilité aux bains publics. Les bains douches ont été abandonnés à Ixelles, rénovés à Saint-Josse, remplacés par des fonctions contemporaines (salles de sport, saunas, jacuzzis, ...) à Schaerbeek... Les Bains de Forest ont perdu leur fonction d'origine et sont occupés par un collectif d'artistes. Les bains de Bruxelles centre sont aujourd'hui les seuls à maintenir des bains douches. Les bains turcs ont été maintenus à Saint-Gilles.

Le programme organisationnel des piscines est particulièrement contraignant puisqu'il impose l'éclairage naturel, la limitation des déperditions de chaleur, un relatif confort acoustique, la résistance à la température et à l'humidité, la sécurité des baigneurs... Les bains constituent un patrimoine fragile du fait de l'exposition à la corrosion et des évolutions des normes d'hygiène et des techniques qui imposent ou justifient des interventions parfois malheureuses sur le plan architectural.

En région bruxelloise, une seule piscine est protégée, l'ancienne piscine du Résidence Palace (ill. 5), complexe d'appartements de luxe construit dans les années vingt. Propriété de l'État, elle est fermée depuis 2002.

\section{5 : La piscine du Résidence Palace}

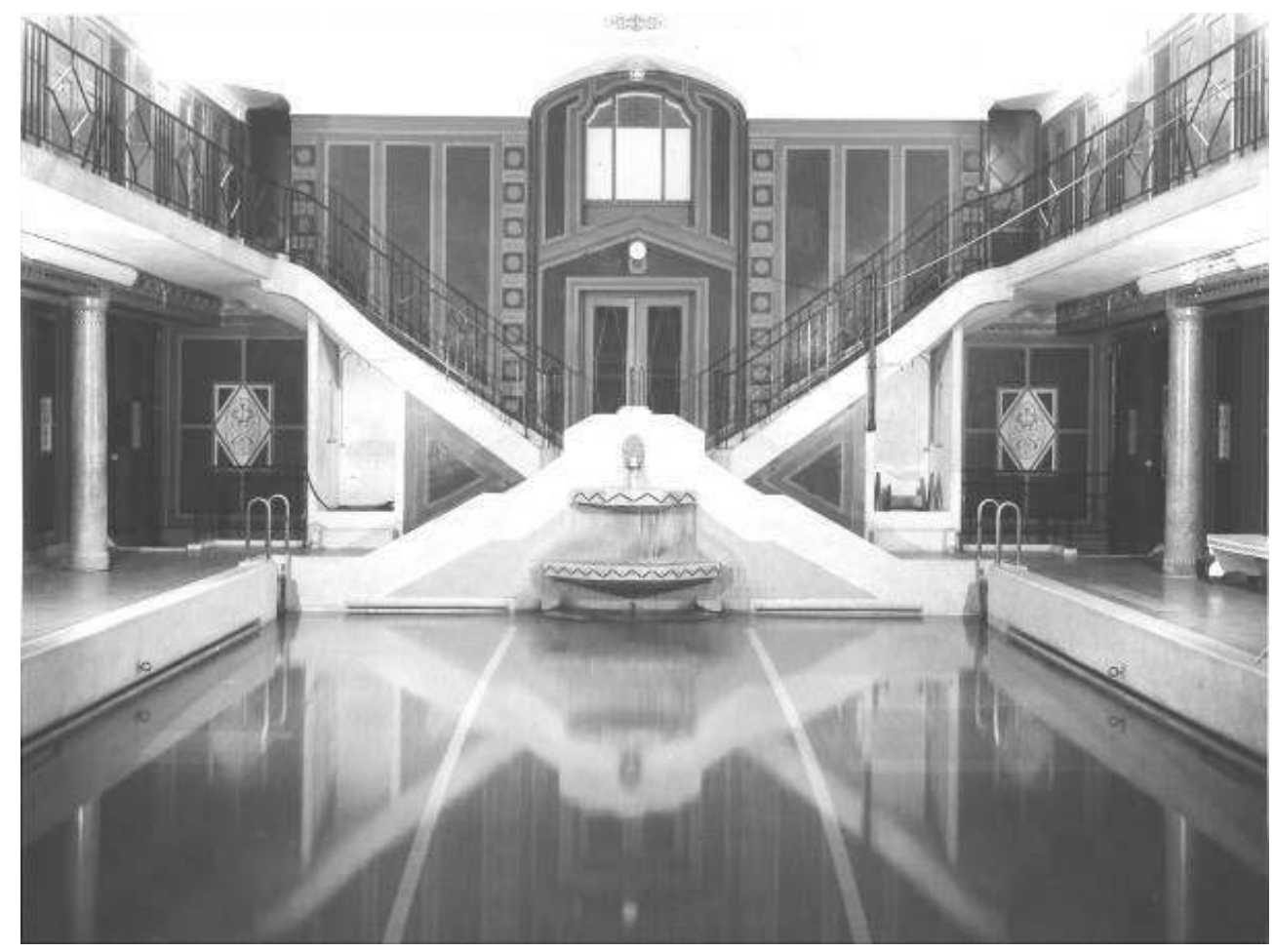

CL. ARCHIVES D'ARCHITECTURE MODERNE, BRUXELLES

Une étude globale des bassins de natation bruxellois a été commandée par la commission royale des monuments et des sites qui devrait contribuer à promouvoir la protection des autres piscines citées dans cet article. 


\section{NOTES}

1. Une étude globale des piscines en région bruxelloise a été réalisée à l'initiative de la commission royale des monuments et des sites : CORDEIRO P., BASYN J.M., PATRICIO T., $E$ tude des bassins de natation situés en Région bruxelloise, 2 vol., 1900-1960 et 1960 à aujourd'hui, non publiée, Bruxelles, février 2006. Elle actualise les travaux de Griet Meyfroots, qui font référence en la matière (voir bibliographie).

2. Une Piscine pour les Marolles, Cent ans de projets, Bains de Bruxelles, Archives de la Ville de Bruxelles, 2004.

3. Notice de présentation des Bains de Saint-Gilles, circa 1905.

4. Ibid.

5. Tiré d'un spectacle de revue, vers 1905. La revue est, en Belgique, une forme de théâtre satirique populaire.

6. Débats au Conseil communal d'Ixelles, cités dans la brochure La Piscine d'Ixelles a cent ans, 1904-2004.

7. Photographie dans l'Émulation, 1907. Le bassin de natation était assorti à un gymnase doté d'une façade monumentale côté rue, qui subsiste, et à des douches individuelles.

8. Victor Boin, « Natation, un sport qu'il faut encourager, il est utile et hygiénique, la question des bassins », La vie moderne, 15 mars 1911.

9. Plans publiés dans L'Émulation, 1880.

10. La Tribune de Bruxelles, 12 novembre 2004.

11. Dixit Jean Paulis, concepteur du Daring-solarium de Molenbeek, aujourd'hui démoli.

12. Georges Dedoyard (1897-1988) fut une figure importante du mouvement moderne à Liège. La piscine de la Sauvenière est publiée dans L'Art de bâtir, août 1942, nº 8.

13. P. Cordeiro, J.M. Basyn, T. Patricio, Étude des bassins de natation situés en Région bruxelloise, 2 vol., 1900-1960 et 1960 à aujourd'hui, non publiée, Bruxelles, février 2006.

14. Géo de Vlaminck (1897-1980) obtint en 1921 le premier prix en composition monumentale au cours de Constant Montald, maître du symbolisme en Belgique. Lors d'un voyage en Italie, il se passionne pour la fresque et la mosaïque qu'il intègrera par la suite dans des réalisations architecturales qui relèvent de l'Art déco. Il a participé aux travaux de l'École de La Cambre, ouverte à Bruxelles en 1926 par Henri Van de Velde, issu du Bauhaus.

15. On peut voir entre autres choses, dans l'atelier de l'artiste admirablement mis en valeur par l'association des Amis de Géo de Vlaminck, l'un des onze cartons préparatoires conservés réalisés pour la mosaïque, des photographies de la mise en œuvre et un petit cabinet de curiosité qui rassemble les coquillages, coraux et hippocampes qui figurent sur la mosaïque.

Géo De Vlaminck a réalisé une autre composition destinée à la piscine de Salzinnes : «Ballet aquatique de poissons poursuivant des naïades autour d'un récif de corail ». Cette fresque peinte a été recouverte d'une couche de peinture en 1994 par l'administration de la ville de Namur, qui gère la piscine.

16. Piscines en ébullition?, Rapport du colloque des 12 et 13 novembre 2004, organisé par les Amis de Géo de Vlaminck. 


\section{RÉSUMÉS}

Avant que la rivière ne fût enterrée et polluée par les industries, les Bruxellois se baignaient dans la Senne. Les premiers établissements de bain, à l'instar du bain Royal (1879), étaient d'initiative privée et réservés à la bourgeoisie urbaine. Au tournant du XIXe et du XXe siècle, les communes des faubourgs industriels ouvrent des bains publics assortis de baignoires et de bains douches dédiés avant tout à des finalités d'ordre hygiéniste et prophylactique. Ces établissements de bains subsistent dans les communes d'Ixelles et de Saint-Gilles. À partir de l'entre-deux-guerres, l'agglomération bruxelloise se dote progressivement de bassins de natation à de fins sportives et de détente. Les contraintes liées à l'hygiène révolutionnent l'organisation des espaces et s'affirment dans le choix des matériaux et d'une esthétique fonctionnelle. C'est le cas des Bains de Saint-Josse (1930-1933), de ceux du centre (1950-1953) ou au Neptunium (1950-1957). Ces piscines constituent des lieux d'un intérêt patrimonial et historique indéniable.

Bains-Baden, swimming pools in Brussels

Before the river got drained and polluted by industries, the inhabitants from Brussels used to bath in the Senne. After the fashion of the "bain Royal" (1879), the first bath houses resulted from private initiative and were used by the urban bourgeoisie. At the turn of the XIX ${ }^{\text {th }}$ century, the suburban industrial towns opened public bath houses with bathtubs and shower rooms with hygienist and prophylactic aims in view. These facilities remain intact in the towns of Ixelles and Saint-Gilles. From the interwar years, Brussels and its suburbs gradually equipped with swimming pools for sport and leisure. Constraints of public health revolutionized the spatial layout and got really noticeable while choosing materials and a functional design. They were so in the "Bains de Saint-Josse" (1930-1933), the "Bains du centre" (1950-1953) and the Neptunium (1950-1957). These swimming pools are definitely significant historic places worthy of preservation.

Bains-Baden, die Brüsseler Badeanstalten

Vor der Überbauung des Flusses und dessen Verschmutzung durch die Industrie badeten die Brüsseler noch in der Senne. Die ersten Badeanstalten wie das Royalbad (1879), die durch private Initiative entstanden, waren den wohlhabenden Stadtbürgern zugänglich. Um die letzte Jahrhundertwende bauten die Gemeinden der industriellen Vororte öffentliche Dusch-und Wannenbäder zu hygienischen und gesundheitsfördernden Zwecken. Solche Badeanstalten existieren noch in den Gemeinden Ixelles und Saint-Gilles. Ab der Zwischenkriegszeit stattete sich die Großstadt Brüssel mit Schwimmbecken für Sport und Erholung aus. Der Bau von Badeanstalten nach hygienischen Vorschriften bietet ein hervorragendes Beispiel funktionaler Ästhetik, die sich in der innovativen Veränderung des Raumkonzepts und der Verwendung zweckmäßiger Materialien ausdrückt. Insofern sind die Bains de Saint-Josse (1930-1933), die Bains du Centre (1950-1953) oder das Neptunium (1950- 1957) Einrichtungen von besonderem historischen und kulturellen Interesse. 
INDEX

Index chronologique : XIXe siècle, XXe siècle, époque contemporaine

Mots-clés : piscine

Keywords : swimming pool

Schlüsselwörter : Schwimmbad

\section{AUTEUR}

\section{ISABELLE PAUTHIER}

Isabelle Pauthier est née en 1968. Après avoir fait ses classes d'hypokhâgne et de khâgne, elle obtient une maîtrise d'histoire à l'université de Nancy II. À Paris, elle suit, parallèlement aux classes préparatoires au concours de l'École du patrimoine à l'École du Louvre puis à la Sorbonne, le séminaire de Jean-Michel Leniaud à l'École pratique des hautes études. Attachée d'éditions auprès de CFC-Éditions à Bruxelles en 1996, elle est directrice de l'Atelier de recherche et d'action urbaines, association sans but lucratif, à Bruxelles, depuis 1997. À ce titre, elle rédige des articles de fonds et des textes de conférences de presse sur les principaux enjeux de l'aménagement urbain à Bruxelles principalement dans les champs du logement, de la mobilité, de la protection du patrimoine, des enjeux du développement économique et de l'évolution institutionnelle de Bruxelles. www.arau.org. Elle est également administratrice d'Inter-Environnement Bruxelles, fédération des comités d'habitants et des Archives d'architecture moderne. 